\title{
Solution Neighbourhoods for Constraint-Directed Local Search
}

\author{
Jun He, Pierre Flener, and Justin Pearson \\ Uppsala University, Department of Information Technology, Uppsala, Sweden \\ Firstname.Lastname@it.uu.se
}

\begin{abstract}
We propose solution neighbourhoods, which contain only solutions to a chosen constraint, as the solutions to a constraint capture the structure of the constraint. We save the time needed for neighbourhood evaluation of that constraint by using a solution neighbourhood. This may be useful especially for constraints for which there exists no known constanttime algorithm for neighbour evaluation. We design a solution neighbourhood for the very useful automaton constraint, and demonstrate the practicality of our approach on a library of nurse scheduling instances. We show the feasibility of designing solution neighbourhoods for other constraints.
\end{abstract}

\section{INTRODUCTION}

In constraint-based local search (CBLS, e.g., [6]), constraints are used to describe and control local search for solving a combinatorial problem. From an initial assignment of values to all decision variables, CBLS iteratively moves to another assignment, which ideally decreases the total amount of constraint violation, by exploring a neighbourhood of small changes to the current assignment, until an assignment with zero constraint violation is found or until allocated resources are exhausted. Meta-heuristics are used to escape local optima. In CBLS, search can be constraintdirected or variable-directed, and it is crucial to choose a good neighbourhood and explore it efficiently. We address how to design a neighbourhood in constraint-directed search.

In constraint-directed search (CDS), first a (possibly most) violated constraint is selected, and then a (possibly best) move is found by exploring a neighbourhood of that constraint, where a (possibly best) move is a move that (possibly maximally) decreases the violation of the whole constraint system. There are at least two ways to design a neighbourhood of a chosen constraint: a variable-directed neighbourhood selects a few decision variables of the constraint, and designs a neighbourhood by changing the assignments to those decision variables; a constraint-directed neighbourhood designs a neighbourhood according to the possible violation changes

Permission to make digital or hard copies of all or part of this work for personal or classroom use is granted without fee provided that copies are not made or distributed for profit or commercial advantage and that copies bear this notice and the full citation on the first page. To copy otherwise, to republish, to post on servers or to redistribute to lists, requires prior specific permission and/or a fee.

SAC'12 March 25-29, 2012, Riva del Garda, Italy.

Copyright 2011 ACM 978-1-4503-0857-1/12/03 ...\$10.00. of the chosen constraint. With a variable-directed neighbourhood, the search focuses on a small and prospective neighbourhood, which is efficient but easily trapped at a local optimum. With a constraint-directed neighbourhood, the search exploits some of the substructure of the combinatorial problem, namely the chosen constraint, but it may be inefficient if the neighbourhood is large. Hence it is interesting to integrate the two methods, and design a hybrid neighbourhood, namely a variable-directed and constraint-directed neighbourhood, so that we can benefit from the advantages of both. We address how to design a hybrid neighbourhood for the very useful automaton constraint [3], a particular case of which is also known as the regular constraint [5].

When designing a hybrid neighbourhood for the automaton constraint, we are interested in a neighbourhood where all neighbours satisfy the constraint, called a solution neighbourhood, for the following reasons: first, it is easy to find a solution to an automaton constraint that is close to a given violating assignment by exploring the structure of the automaton constraint; second, as far as we know, the best algorithm for neighbour evaluation of the automaton constraint takes time linear in its number of decision variables [4], which is expensive, thus we can avoid such an expensive neighbour evaluation by using a solution neighbourhood.

There is of course a cost of designing a solution neighbourhood. Usually, neighbourhoods are generated in constant time per neighbour, and a neighbour evaluation takes constant time for many constraints, but it (necessarily) takes more than constant time for some constraints; e.g., the automaton constraint. We investigate the following question: when does it pay off to generate a small neighbourhood of solutions to a given constraint, in non-constant time per neighbour, so as to achieve evaluation in zero time of these neighbours for that constraint? We show that such an investment in neighbourhood generation is well amortised for such expensive constraints as automaton, and that even some cheap constraints such as alldifferent can benefit from this idea.

The contributions and organisation of this paper are as follows: we introduce the idea of using a hybrid neighbourhood for CDS in Section 2; we show how to design a solution neighbourhood for the automaton constraint in Section 3, where all neighbours are solutions to the chosen constraint and introduce useful diversification (in addition to any diversification introduced by a meta-heuristic); we present experiment results establishing the practicality of our method in Section 4; finally, in Section 5, we show the feasibility of designing solution neighbourhoods for other constraints, summarise this work, and discuss related and future work. 


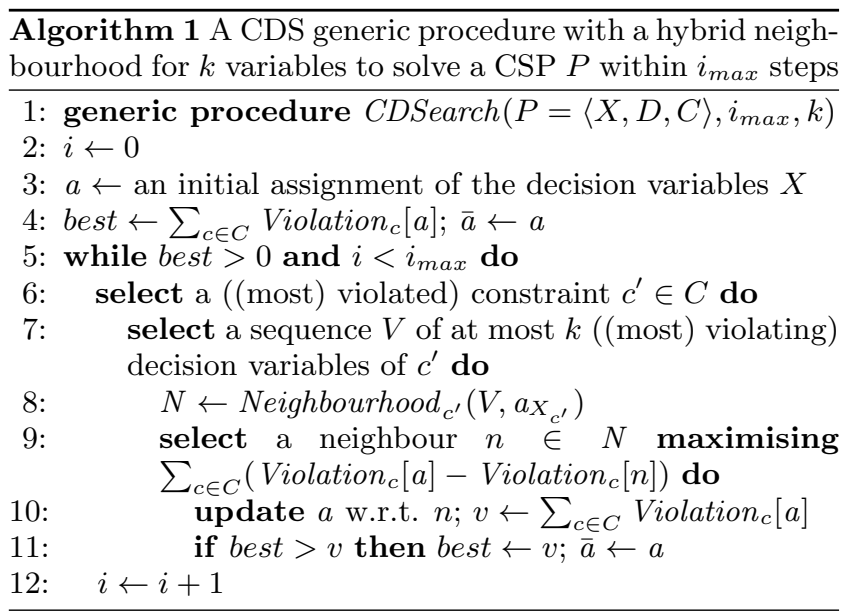

\section{CDS WITH A HYBRID NEIGHBOURHOOD}

Let $P=\langle X, D, C\rangle$ denote a constraint satisfaction problem (CSP), where $X$ is a finite sequence of decision variables, $D$ is the sequence of domains of $X$, and $C$ is a finite set of constraints; let $a$ be the current assignment of $X$; for any constraint $c \in C$, let $X_{c} \subseteq X$ be the decision variables involved in $c$; and let $a_{X_{c}}$ be the current assignment of $X_{c}$. In a CBLS system (such as the CBLS back-end of COMET [6]), two data structures are automatically maintained incrementally: Violation $n_{c}[a]$ denotes the violation of constraint $c$ under the assignment $a$; Violation $n_{c}[x, a]$ denotes the violation of decision variable $x$ in constraint $c$ under the assignment $a$. We call $\sum_{c \in C}$ Violation $_{c}[a]$ the system violation and $\sum_{c \in C}$ Violation $_{c}[x, a]$ the system variable violation of decision variable $x$ under assignment $a$.

The generic procedure $C D \operatorname{Search}\left(P, i_{\max }, k\right)$ in Algorithm 1 describes how $P$ is solved in CDS with a hybrid variabledirected (line 7) and constraint-directed (line 8) neighbourhood. The choice of parameter $k$ is problem-specific; we will address this issue for the experiments in Section 4.2.1. It first initialises the iteration counter $i$ to zero (line 2), a to an initial assignment (line 3), best (which denotes the violation of the current best assignment) to the violation of the initial assignment, and $\bar{a}$ (which denotes the current best assignment) to the initial assignment (line 4). It then checks the termination condition (either a solution has been found or the maximum number $i_{\max }$ of iterations has been reached) of the search (line 5). If the termination condition is not met, then it selects a ((most) violated) constraint $c^{\prime} \in C$ (line 6) and a sequence $V$ of at most $k$ ((most) violating) decision variables of $c^{\prime}$ (line 7 ), and then designs a hybrid variable-directed (by $V$ ) and constraint-directed (by $c^{\prime}$ ) neighbourhood $N$ under the current assignment $a$ by calling the function Neighbourhood ${ }_{c^{\prime}}\left(V, a_{X_{c^{\prime}}}\right)$ (line 8), which returns a neighbourhood obtained by reassigning $V$, an instance for the automaton constraint being described in Algorithm 2 and discussed in Section 3.2.1. Each neighbour $n \in N$ is evaluated by the sum of the differentially computed violation changes over all constraints $c \in C$ upon the corresponding move (line 9), and a randomly chosen best move achieving the maximum violation decrease for $C$ is made (line 10). If a new best assignment is found upon the move, then best and $\bar{a}$ are updated (line 11). The iteration counter $i$ is increased after each iteration (line 12).

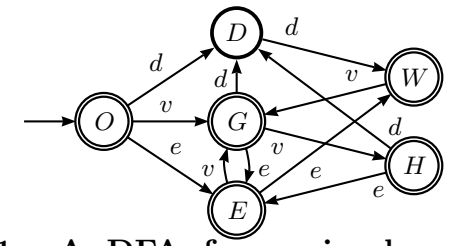

Figure 1: A DFA for a simple work scheduling constraint for one employee

Algorithm 1 lacks meta-heuristics to escape local optima, as these are orthogonal issues that can be addressed separately [6]. Diversification and intensification are not discussed here for the same reason, because we make no assumptions on the assignment $a$. Note that each constraint has its own neighbourhood generator based on the implementation of the function Neighbourhood ${ }_{c}\left(V, a_{X_{c}}\right)$. This can be implemented as an API extension to the constraint class of some CBLS system, such as the CBLS back-end of COMET [6] (available at dynadec.com).

\section{SOLUTION NEIGHBOURHOODS}

After an introduction to the automaton constraint, we show how to design a solution neighbourhood for the automaton constraint. We only consider the automaton constraint without counters here, but plan to expand on this.

\subsection{The Automaton Constraint}

The automaton constraint is defined as automaton $(X, M)$, where $X$ is a sequence of decision variables, and $M$ is a deterministic finite automaton (DFA). A DFA is a tuple $\left\langle Q, \Sigma, \delta, q_{0}, F\right\rangle$, where $Q$ is a finite set of states, $\Sigma$ is the alphabet, $\delta: Q \times \Sigma \rightarrow Q$ is the transition function, $q_{0} \in Q$ is the start state, and $F \subseteq Q$ is the set of accepting states. The automaton constraint is satisfied iff $M$ accepts the word made of the values of the sequence $X$ of decision variables.

For example, Figure 1 gives a DFA that describes a work scheduling constraint for one employee. There are values for two work shifts, namely day $(d)$ and evening $(e)$, and a value for enjoying a day of vacation $(v)$. The set of words accepted by this DFA defines the set of acceptable shift sequences.

Given an automaton $(X, M)$ constraint with $|X|=n$ decision variables and the DFA $M=\left\langle Q, \Sigma, \delta, q_{0}, F\right\rangle$, the (minimised) conjunctive product of $M$ with the DFA accepting $\Sigma^{n}$ gives a DFA $M^{\prime}$ that only accepts $n$-letter words in the language of $M$. This construction is known in CP as the unrolling of $M$ for words of length $n$ [5]. For example, the DFA $M^{\prime}$ obtained by unrolling $M$ in Figure 1 for words of length $n=6$ is in Figure 2 (the reader can ignore the different line styles of the transitions and states at the moment). Note that any state $q_{m}$ of $M$ in vertical layer $j$ is named $q_{m}^{j}$ in $M^{\prime}$. Any assignment made of the values labelled on the arcs of a path in $M^{\prime}$ is a solution to the automaton constraint, and vice versa. Note that whenever a path in $M^{\prime}$ is mentioned, we mean a path from the start state of $M^{\prime}$ to any accepting state of $M^{\prime}$. In Figure 2, there are 49 such paths, hence the automaton $(X, M)$ constraint with $|X|=n=6$ decision variables and the DFA $M$ in Figure 1 has 49 solutions among the $|\Sigma|^{n}=3^{6}=729$ possible assignments. By an abuse of language, we sometimes refer to an assignment as a path, such that the assignment is made of the values labelled on the arcs of the path.

As far as we know, there are two versions of violation measure [4], based on Hamming distance, for the automaton 


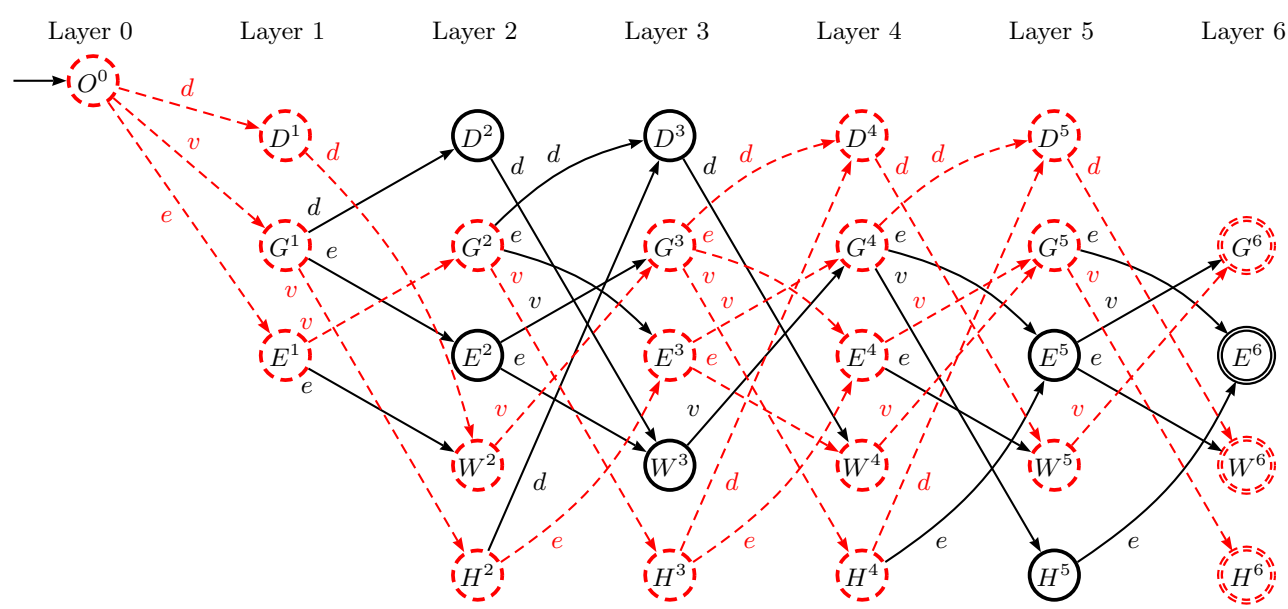

Figure 2: The DFA $M^{\prime}$ obtained by unrolling the DFA $M$ in Figure 1 for words of length 6 . State $q_{m}$ of $M$ in layer $j$ is named $q_{m}^{j}$ in $M^{\prime}$. Given the current assignment $a=\langle d, v, v, e, d, v\rangle$ to the decision variables $X=\left\langle X_{1}, \ldots, X_{6}\right\rangle$, and the sequence $V=\left\langle X_{1}, X_{4}\right\rangle$ of $k=2$ decision variables chosen to be reassigned for designing a neighbourhood, the resulting neighbourhood contains all the 7 dashed paths.

constraint in CBLS. We use the one that is shown in [4] to work both theoretically and experimentally much better.

\subsection{Designing a Solution Neighbourhood}

Consider an automaton $(X, M)$ constraint with a current assignment $a$ to the decision variables $X$, a sequence $V \subseteq X$ of decision variables to be reassigned for designing a solution neighbourhood, and the DFA $M^{\prime}$ obtained by unrolling $M$ for words of length $|X|$. With the structure of solutions to the automaton constraint as described in Figure 2, topological sorting is used, as in Algorithm 2, to design efficiently a solution neighbourhood for the automaton constraint.

\subsubsection{Description of the Algorithm}

Algorithm 2 with the unique parameter $|V|=k$ works by dashing some states and transitions in the DFA $M^{\prime}$ obtained by unrolling the DFA $M$ for words of length $|X|$ (lines 2 to 11), so that every dashed path in $M^{\prime}$ (except the current assignment $a$ ) denotes a neighbour in the neighbourhood; and it returns the neighbourhood containing all dashed paths except $a$ in $M^{\prime}$ (line 12). When considering decision variable $X_{i}$ and visiting a dashed state $q_{m}^{i-1}$ in $M^{\prime}$ : if $X_{i}$ is in $V$, then all outgoing transitions from state $q_{m}^{i-1}$ and their target states in $M^{\prime}$ are dashed (lines 5 to 7 ), so that all feasible assignments (in the sense that a dashed path can be extended) to decision variable $X_{i}$ are included in the neighbourhood; otherwise Algorithm 2 tries to extend a dashed path by preserving the assignment of $X_{i}$ (lines 8 and 9), or (at failure to do so; line 10) by choosing randomly one outgoing transition from $q_{m}^{i-1}$, hence providing the chance to change also the assignment of $X_{i} \notin V$ (line 11; this introduces more diversity, so as to stop the search easily being trapped in some local optimum). As all transitions and states not leading to an accepting state are by construction not in $M^{\prime}$, there is a path to an accepting state of $M^{\prime}$ passing each state of $M^{\prime}$. Hence for each dashed state (in lines 2, 7, 9, and 11), Algorithm 2 always finds such a path passing this dashed state. As any path in $M^{\prime}$ is a solution to the automaton constraint, Algorithm 2 designs a solution neighbourhood for the automaton constraint.

For example, in Figure 2 with the current assignment $a=\langle d, v, v, e, d, v\rangle$ to $|X|=6$ decision variables and a se- $\overline{\text { Algorithm } 2 \text { Design a solution neighbourhood directed by }}$ an automaton $(X, M)$ constraint and a sequence $V \subseteq X$ of decision variables under the current assignment $a$ to the decision variables $X$, where the DFA $M^{\prime}$ obtained by unrolling the DFA $M$ for words of length $|X|$ is pre-computed.

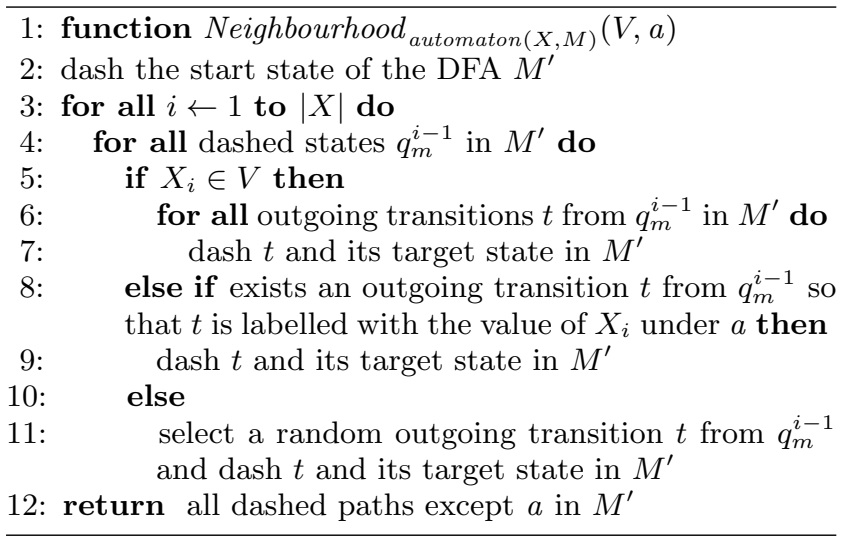

quence $V=\left\langle X_{1}, X_{4}\right\rangle$ of $k=2$ decision variables, the resulting neighbourhood contains all assignments related with any dashed path in $M^{\prime}$. There are 7 dashed paths, hence the resulting neighbourhood has 7 neighbours among the 49 solutions. Algorithm 2 first dashes the start state $O^{0}$ in layer 0 of $M^{\prime}$ (line 2). Then it dashes all outgoing transitions from $O^{0}$ and their target states $\left(D^{1}, E^{1}\right.$, and $\left.G^{1}\right)$ in layer 1 , as $X_{1} \in V$ (lines 5 to 7 ). Next it visits the dashed states $D^{1}$, $E^{1}, G^{1}$ in this order: when visiting $D^{1}$, as there is no outgoing transition from $D^{1}$ that is labelled with $v$ (the value assigned to $X_{2}$ under $a$ ), the only outgoing transition from $D^{1}$ and its target state $W^{2}$ in layer 2 are dashed (line 11); when visiting $E^{1}$, as there is an outgoing transition from $E^{1}$ that is labelled with $v$, this transition and its target state $G^{2}$ in layer 2 are dashed (lines 8 and 9); when visiting $G^{1}$, as there is an outgoing transition from $G^{1}$ that is labelled with $v$, this transition and its target state $H^{2}$ in layer 2 are dashed (lines 8 and 9). The process continues for a while, then Algorithm 2 will visit $E^{4}$ in layer 4 . As there is no outgoing transition from $E^{4}$ that is labelled with $d$ (the value 
assigned to $X_{5}$ under $a$ ), one (and only one) random transition between the two outgoing transitions $\left(E^{4} \stackrel{v}{\longrightarrow} G^{5}\right.$ and $E^{4} \stackrel{e}{\longrightarrow} W^{5}$ ) will be chosen to be dashed (in Figure 2, the transition $E^{4} \stackrel{v}{\longrightarrow} G^{5}$ is chosen). The process continues until all dashed states are visited, and returns the neighbourhood of 7 neighbours (7 dashed paths on Figure 2).

\subsubsection{Complexity and Theoretical Evaluation}

To establish the time complexity of Algorithm 2, let $M=$ $\left\langle Q, \Sigma, \delta, q_{0}, F\right\rangle$ be the DFA of an automaton $(X, M)$ constraint with $|X|=n$ decision variables, let all decision variables $x \in X$ have the same domain $\Sigma$, let $M^{\prime}$ denote the DFA obtained by unrolling $M$ for words of length $n$, and let a sequence $V \subseteq X$ of $k$ decision variables be chosen to design a solution neighbourhood. Lines 3 to 11 explore all transitions and states in $M^{\prime}$ at most once, thus take $O(n \cdot|\delta|+n \cdot|Q|)$ time (since $M^{\prime}$ has $O(n \cdot|\delta|)$ transitions and $O(n \cdot|Q|)$ states); line 12 explores all dashed paths in $M^{\prime}$ once, thus takes $O(n \cdot|N|)$ time (every path has a length of $n$ ), where $|N|$ is $O\left(|\Sigma|^{k}\right)$, since for each reassignment of $V$, at most one path will be found and inserted into the neighbourhood. Hence Algorithm 2 takes $O\left(n \cdot\left(|\delta|+|Q|+|\Sigma|^{k}\right)\right)$ time in total.

Compared with designing a pure variable-directed neighbourhood, which takes $\Theta\left(|\Sigma|^{k}\right)$ time (designing a neighbourhood of size $|\Sigma|^{k}-1$ ), our method of designing a solution neighbourhood for the automaton constraint takes $n$ times more time. However we do not need to evaluate the violation changes for the chosen automaton constraint when exploring the solution neighbourhood, as the violation changes for that automaton constraint are all the same (the solution neighbourhood contains only solutions to that automaton constraint) and make no contribution to distinguishing the neighbours. Note that the best known algorithm for differential neighbour evaluation for the automaton constraint on $n$ decision variables takes $O(n)$ time [4], which is expensive. Hence the time cost of designing the solution neighbourhood with large enough $k$ will be paid off by the time needed for exploring the neighbourhood of the automaton constraint.

Consider the frequent problem pattern with at least two automaton constraints, each on $n$ decision variables, so that there is no sharing of decision variables between any two automaton constraints. A search directed by the automaton constraints normally takes $O(n)$ time per neighbour (of the chosen automaton constraint) for differentially evaluating the impact on all the automaton constraints. The benefit of our proposal is that this cost can be brought down to zero time per neighbour (the time of evaluating the impact on any constraints that share decision variables with the chosen automaton constraint is unchanged), by investing the cost of designing a solution neighbourhood instead. Usually, the saving of $O(n)$ time per neighbour amortises that investment. We study a rostering problem of this pattern in Section 4. In Section 5, we show that even constraints with differential neighbour evaluation in $O(1)$ time can benefit from a solution neighbourhood.

\section{EXPERIMENTAL EVALUATION}

We now investigate experimentally the practicality of the proposed CDS with a solution neighbourhood, by comparing it to a non-hybrid neighbourhood, here called the differ-byone neighbourhood, that contains all assignments that differ from the current assignment in any decision variable of a chosen constraint is explored at each iteration. Note that the differ-by-one neighbourhood is much larger than a variable-directed neighbourhood, which contains neighbours obtained by only changing the assignment of one chosen decision variable of a chosen constraint, and the differ-by-one neighbourhood usually works better for CDS. We implemented Algorithm 2 for the CBLS back-end of Comet [6] and ran experiments on the benchmark of NSPlib [7].

Note that the objective of our experiments is not to beat the state of the art of nurse rostering, but to show that using a solution neighbourhood can speed up CDS without re-tuning the meta-heuristics.

\subsection{The Problem and the Model}

NSPlib [7] is a very large repository of (artificially generated) instances of the nurse scheduling problem (NSP), which is about constructing a duty roster for nursing staff. Let $N$ be the number of nurses, $D$ the number of days of the scheduling horizon, and $S$ the number of shifts. The objective is to construct an $N \times D$ matrix of values in the integer interval $[1, \ldots, S]$, with value $S$ representing the off-duty shift.

In instance files, there are hard coverage constraints and soft preference constraints; we only use the former (as optimisation is orthogonal to our searching concerns), which can be modelled by atLeast constraints on the columns. There are instance files for $30 \times 28$ and $60 \times 28$ rosters.

In case files, there are hard constraints on the rows. We model all the constraints on a row with a single automaton constraint. There are 8 case files for the $N \times 28$ rosters.

COMET has the atLeast constraint as a built-in; however its definition of the violation of decision variables does not work well for our experiments. In the COMET (current version 2.1.1) implementation of the atLeast constraint, variable violations can be non-zero even if the atLeast constraint is satisfied; this may mislead the search if variable violations are used. We coded a revised atLeast constraint for our experiments, where the violations of all decision variables are zero iff the violation of the atLeast constraint is zero. Our experiments (not reported here for space reasons) show that our revised atLeast constraint works up to 5 times faster (and never slower) than the built-in one on our search procedures for the NSPlib problem.

The automaton constraint is not a built-in of the CBLS back-end of COMET; we implemented it as in [4].

\subsection{The Search Procedures}

Two search procedures were tried for the NSPlib problem.

\subsubsection{CDS with a Solution Neighbourhood}

We instantiate as follows the CDS generic procedure with a hybrid neighbourhood described in Algorithm 1.

When selecting a most violated constraint (line 6 of Algorithm 1), a constraint with the maximum constraint violation is usually selected; however, in our experiments, we observed that selecting a constraint that has the maximum sum of the system variable violations of the decision variables in the constraint works up to 3 times better (and never slower) than selecting a constraint with the maximum constraint violation (these experiments are not reported here for space reasons). We also observed that always selecting a most violated automaton constraint works up to 10 times faster (and never slower) than selecting a most violated constraint among all automaton and atLeast constraints. 
When selecting $|V|=k$ decision variables to design a solution neighbourhood (line 7), we first select a random violating decision variable, and then select $k-1$ random decision variables. We observed that this always works up to 7 times faster than selecting the $k$ most violating decision variables, and that this works up to 2 times faster than selecting $k$ random decision variables (these experiments are not reported here for space reasons).

There are two ways to determine the value of $k$ : (1) Choosing a static value. We experimented with $k=1$ until $k=4$ to avoid designing a too large neighbourhood. Note that every decision variable in NSPlib has the same domain of $S=4$ values, thus a solution neighbourhood has a maximum size of $S^{k}=4^{4}=256$ for $k=4$. (2) Choosing a value dynamically during search. We experimented with $k=1$ initially; if a better assignment cannot be found in $k^{2} \cdot 2^{k-1}$ iterations (experimentally determined threshold; details are omitted for space reasons) and $k<4$, then we increase the value of $k$ by one. We never decrease the value of $k$, as we did not find a good way yet of doing it. We give a comparison of the two methods in Section 4.3.

Our chosen meta-heuristic is tabu search with restarts. The length of the tabu list is the maximum between 6 and the sum of the violations of all constraints. The best assignment so far is maintained. Restarting is done every $N \cdot D$ iterations. The expression for the length of the tabu list and the restart criterion were experimentally determined; details are omitted for space reasons.

\subsubsection{Classical CDS}

In [4], a variable-directed search is used to solve the NSPlib problem. However, we can achieve an improvement by a factor of up to 50 over [4] by replacing its variable-directed search with constraint-directed search, where a most violated automaton constraint is selected at each iteration (as in Section 4.2.1), the differ-by-one neighbourhood of the constraint is explored, and a best move that maximally decreases the system violation is made. Our chosen metaheuristic is tabu search with restarts (as in Section 4.2.1).

\subsection{The Results}

In Table 1, we give a comparison of the two search procedures in Sections 4.2.1 and 4.2.2 on NSPlib instances [7], except for those known from [2] to be unsatisfiable (in order to accelerate the experiments). Other instances might be unsatisfiable (this information cannot be extracted from the NSPlib website), hence the percentage of solved instances might not reach $100 \%$ for that reason.

All experiments were run under Comet (version 2.1.1) and Suse Linux 11.3 on a $3.07 \mathrm{GHz}$ Intel Core i7 with a 3GB RAM. All runs were allocated $30 \mathrm{CPU}$ seconds, and the average performance was recorded for each instance over 25 runs. For each run of an instance, all search procedures started with the same randomly generated initial assignment. We did not run instances of cases 1 to 10 and cases 12 to 14 , as they are very easy [2]; we also did not run instances of case 11 , as the DFAs (30 or 60 DFAs with 11816 states and 41922 transitions each, before unrolling) for these instances are too large for handling by the automaton constraint of [4] under Comet (version 2.1.1) on the chosen hardware. Note that this memory problem is independent of search, hence both our search procedures in Sections 4.2.1 and 4.2.2 suffer from the same memory problem.
In Table 1, the average performance over 25 runs each of selected instances (namely 10 instances each for all combinations of the NSPlib complexity indicators) for each case and $N$ is given. Each row first specifies the instances (denoted by Case, $N$, and Instances), and then gives the performance of each search procedure, namely the average percentage of instances solved without timing out (denoted by \%S), the average runtime in seconds (denoted by Sec), and the average number of iterations (denoted by Iter). Numbers that denote the best performance in each row are boldfaced, and runtimes with a margin of $5 \%$ of the best performance in each row are also boldfaced. There are four rows for each case and $N$ : the first one denotes the average performance over all selected instances; the following three denote the performance of three hand-picked instances, which represent three difficulty levels with the order of difficulty increasing.

Comparing the two methods mentioned in Section 4.2.1 for choosing the value of $k$, we observed that: (1) Considering the average percentage of solved instances and the average runtime, the method with dynamic $k$ and the method with $k=4$ are the winners. (2) Considering the average number of iterations, the method with $k=4$ always wins, as it explores the largest neighbourhood. (3) Considering the three difficulty levels among the selected instances, the method with $k=1$ always wins for solving the easy instances, but never works for the harder instances; the method with $k=4$ and the method with dynamic $k$ always win for solving the harder instances; the method with dynamic $k$ works well among all the three difficulty levels.

Comparing the two search procedures in Sections 4.2.1 and 4.2 .2 , we observed that the search procedure with a solution neighbourhood in Section 4.2.1, except for those with static $k=1$ and $k=2$, always beats the search procedure in Section 4.2.2: considering the average runtime over all selected instances, our method can be up to 2 times faster; considering the runtime of one particular hard instance, our method can be up to 40 times faster.

\section{CONCLUSION}

In summary, we have shown that the idea of using a solution neighbourhood can be useful for constraint-directed local search. We have demonstrated the idea of designing a solution neighbourhood for the very useful automaton constraint. Our experiment results show that our approach not only outperforms the described improvement of [4] on the NSPlib problem, but also outperforms any other CDS procedure that we have tried. Note again that the objective of our experiments was not to beat the state of the art of nurse rostering; thus we do not compare with the large amount of related literature on (meta-)heuristics, because most of it is orthogonal to our work on neighbour evaluation [6].

Three constraint-directed neighbourhoods are introduced in [1], namely the decreasing neighbourhood, preserving neighbourhood, and increasing neighbourhood, which contain neighbours that decrease, preserve, and increase the constraint violation individually upon making the move. Differently from [1], we are here interested in a non-increasing neighbourhood where all neighbours satisfy the constraint. There is of course a cost of designing a solution neighbourhood for a chosen constraint, however our experiment results show that this cost for the automaton constraint is justified by its benefits. For some other constraints, there are ways to design a solution neighbourhood. For example, consi- 
der the alldifferent $(X)$ constraint, where $X=\left\langle X_{1}, \ldots, X_{5}\right\rangle$ is a sequence of 5 decision variables with the same domain $\{1, \ldots, 5\}$. Given an assignment $a=\langle 1,3,5,4,5\rangle$ with a sequence $V=\left\langle X_{3}\right\rangle$ of $k=1$ chosen decision variable, we can design a solution neighbourhood for the alldifferent $(X)$ constraint as follows: we try each possible reassignment of $V$; if a solution to the constraint is obtained (e.g., with $X_{3}=2$ ), then the solution is added to the neighbourhood; otherwise (e.g., with $X_{3}=4$ ), we try to find a solution by further changing the assignment of some decision variables not in $V$ (e.g., with $X_{4}=2$ ), and add the solution (if found) to the neighbourhood. We did experiments with the magic square problem, where the alldifferent constraint is used, and our experiment results (not reported here for space reasons) show that the cost of designing a solution neighbourhood for the alldifferent constraint is also justified by a runtime speed up of more than 3 times for instances of size larger than $5 \times 5$. Note that we do not force the use of a solution neighbourhood for every constraint, as each constraint has its own neighbourhood as described in the generic procedure of Algorithm 1; but we argue that using a solution neighbourhood for some constraints can be helpful.

Consider the connectedness of solution neighbourhoods. Given a constraint $c(X)$ over a sequence of $n$ decision variables $X$ and its solution neighbourhood generator, assume that if there is a solution to $c$ extending the current assignment of decision variables $\left\langle X_{1}, \ldots, X_{i}\right\rangle(1 \leq i<n)$, then the generator can find a neighbour of the current assignment by preserving the assignment of $\left\langle X_{1}, \ldots, X_{i}\right\rangle$. This is the case for the automaton and alldifferent constraints. From an arbitrary initial assignment, any solution $s$ to $c$ can be reached within at most $\left\lceil\frac{n}{k}\right\rceil$ moves by moving closer to $s$ (changing the assignment of $X_{1}$ until $X_{n}$ ) iteratively. As any solution to a CSP must be a solution to each constraint in the problem, we have that any solution to the problem is reachable, and solution neighbourhoods are connected.

Future work includes designing solution neighbourhoods for the automaton constraint with counters and other constraints.

\section{Acknowledgements}

The authors are supported by grant 2007-6445 of the Swedish Research Council (VR), and Jun He is also supported by grant 2008611010 of China Scholarship Council and National University of Defence Technology. Many thanks to the anonymous referees.

\section{REFERENCES}

[1] M. Ågren, P. Flener, and J. Pearson. Revisiting constraintdirected search. Inf. Comput., 207(3):438-457, 2009.

[2] N. Beldiceanu, M. Carlsson, P. Flener, and J. Pearson. On matrices, automata, and double counting. In CP-AI-OR'10, LNCS 6140, pages 10-24. Springer, 2010.

[3] N. Beldiceanu, M. Carlsson, and T. Petit. Deriving filtering algorithms from constraint checkers. In $C P^{\prime} 04, L N C S 3258$, pages 107-122. Springer, 2004.

[4] J. He, P. Flener, and J. Pearson. An automaton constraint for local search. Fundam. Inform., 107(2-3):223-248, 2011.

[5] G. Pesant. A regular language membership constraint for finite sequences of variables. In $C P^{\prime} 04, L N C S 3258$, pages 482-495. Springer, 2004.

[6] P. Van Hentenryck and L. Michel. Constraint-Based Local Search. The MIT Press, 2005.

[7] M. Vanhoucke and B. Maenhout. On the characterization and generation of nurse scheduling problem instances. Eur. J. Oper. Res., 196(2):457-467, 2009. 